\title{
On Regularized Solution for BBGKY Hierarchy of One-Dimensional Infinite System
}

\author{
Tatiana V. RYABUKHA \\ Institute of Mathematics of NAS of Ukraine, 3 Tereshchenkivs'ka Str., Kyiv-4, 01601 Ukraine \\ E-mail: vyrtum@imath.kiev.ua
}

Received October 31, 2005, in final form April 26, 2006; Published online May 14, 2006

Original article is available at http://www.emis.de/journals/SIGMA/2006/Paper053/

\begin{abstract}
We construct a regularized cumulant (semi-invariant) representation of a solution of the initial value problem for the BBGKY hierarchy for a one-dimensional infinite system of hard spheres interacting via a short-range potential. An existence theorem is proved for the initial data from the space of sequences of bounded functions.
\end{abstract}

Key words: BBGKY hierarchy; cumulant; regularized solution

2000 Mathematics Subject Classification: 82C05; 82C40

\section{Introduction}

Last ten years new functional-analytical methods of investigation of the initial value problem to the BBGKY hierarchy for infinite systems have been developed in the books $[1,2,3,4]$. As is well known when constructing a solution of the BBGKY hierarchy of such systems, considerable analytical difficulties arise [5, 6, 7, 8]. Until recently, existence theorems have been proved for one-dimensional systems in the case of a certain class of short-range interaction potentials and three-dimensional case only for hard spheres systems [1].

While constructing the solution of the initial value problem for the BBGKY hierarchy of the classical systems of particles with the initial data from the space of sequences of bounded functions, one is faced with certain difficulties related to divergence of integrals with respect to configuration variables in each term of an expansion of the solution [5] (see also [1, 2]). The same problem arises also in the case of the cumulant representation of the solution stated in [9].

In this paper, we propose a regularization method for the solution of the BBGKY hierarchy in the cumulant representation. Due to this method, the structure of the solution expansions guarantees the mutual compensation of the divergent integrals in every term of the series. We establish convergence conditions for the series of the solution and prove an existence theorem of a local in time weak solution of the BBGKY hierarchy for the initial data from the space of sequences of functions which are bounded with respect to the configuration variables and exponentially decreasing with respect to the momentum variables.

\section{Initial value problem for BBGKY hierarchy}

Let us consider a one-dimensional system of identical particles (intervals with length $\sigma$ and unit mass $m=1$ ) interacting as hard spheres via a short range pair potential $\Phi$. Every particle $i$ is characterized by phase coordinates $\left(q_{i}, p_{i}\right) \equiv x_{i} \in \mathbb{R} \times \mathbb{R}, i \geq 1$. For the configurations $q_{i} \in \mathbb{R}^{1}$ of such a system ( $q_{i}$ is the position of the center of the $i$ th particle), the following inequalities must be satisfied: $\left|q_{i}-q_{j}\right| \geq \sigma, i \neq j \geq 1$. The set $W_{n} \equiv\left\{\left\{q_{1}, \ldots, q_{n}\right\} \mid \exists(i, j), i \neq j \in\{1, \ldots, n\}\right.$ : $\left.\left|q_{i}-q_{j}\right|<\sigma\right\}$ defines the set of forbidden configurations in the phase space of a system of $n$ 
particles. The phase trajectories of such hard sphere system are determined almost everywhere in the phase space $\left\{x_{1}, \ldots, x_{n}\right\} \in \mathbb{R}^{n} \times\left(\mathbb{R}^{n} \backslash W_{n}\right)$, namely, outside a certain set $\mathcal{M}_{n}^{0}$ of the Lebesgue measure zero [1]. The initial data $\left\{x_{1}, \ldots, x_{n}\right\} \in \mathbb{R}^{n} \times\left(\mathbb{R}^{n} \backslash W_{n}\right)$ belong to the set $\mathcal{M}_{n}^{0}$ if (a) there is more than one pair collision at the same moment of time $t \in(-\infty,+\infty)$ or (b) infinitely many collisions occur within a finite time interval.

We assume that the interaction between the hard spheres is given by a potential $\Phi$ with a finite range $R$ such that the following conditions are satisfied:

(a) $\Phi \in C^{2}([\sigma, R]), \quad 0<\sigma<R<\infty$,

(b) $\Phi(|q|)= \begin{cases}+\infty, & |q| \in[0, \sigma), \\ 0, & |q| \in(R, \infty),\end{cases}$

(c) $\Phi^{\prime}(\sigma+0)=0$.

We note that conditions (1) imply the estimate

$$
\left|\sum_{i<j=1}^{n} \Phi\left(q_{i}-q_{j}\right)\right| \leq b n, \quad b \equiv \sup _{q \in[\sigma, R]}|\Phi(q)|\left(\left[\frac{R}{\sigma}\right]\right),
$$

where $\left[\frac{R}{\sigma}\right]$ is the integer part of the number $\frac{R}{\sigma}$.

The evolution of states of the system under consideration is described by the initial value problem for the BBGKY hierarchy $[1,2]$

$$
\begin{aligned}
\frac{\partial}{\partial t} F_{s}\left(t, x_{1}, \ldots, x_{s}\right)= & \left\{H_{s}, F_{s}\left(t, x_{1}, \ldots, x_{s}\right)\right\} \\
& +\int d x_{s+1}\left\{\sum_{i=1}^{s} \Phi\left(q_{i}-q_{s+1}\right), F_{s+1}\left(t, x_{1}, \ldots, x_{s+1}\right)\right\} \\
& +\sum_{i=1}^{s} \int_{0}^{\infty} d P P \cdot\left(F_{s+1}\left(t, x_{1}, \ldots, x_{s}, q_{i}+\sigma, p_{i}-P\right)\right. \\
& -F_{s+1}\left(t, x_{1}, \ldots, q_{i}, p_{i}-P, \ldots, x_{s}, q_{i}-\sigma, p_{i}\right) \\
& +F_{s+1}\left(t, x_{1}, \ldots, x_{s}, q_{i}-\sigma, p_{i}+P\right) \\
& \left.-F_{s+1}\left(t, x_{1}, \ldots, q_{i}, p_{i}+P, \ldots, x_{s}, q_{i}+\sigma, p_{i}\right)\right), \\
\left.F_{s}\left(t, x_{1}, \ldots, x_{s}\right)\right|_{t=0} & =F_{s}\left(0, x_{1}, \ldots, x_{s}\right), \quad s \geq 1,
\end{aligned}
$$

where $\{\cdot, \cdot\}$ is the Poisson bracket, $H_{s}$ is the Hamiltonian of the $s$ particle system, and $F(0)=$ $\left(1, F_{1}\left(0, x_{1}\right), \ldots, F_{s}\left(0, x_{1}, \ldots, x_{s}\right), \ldots\right)$ is a sequence of initial $s$-particle distribution functions $F_{s}\left(0, x_{1}, \ldots, x_{s}\right)$ defined on the phase space $\mathbb{R}^{s} \times\left(\mathbb{R}^{s} \backslash W_{s}\right)$.

Consider the initial value problem for the BBGKY hierarchy (3), (4) with the initial data $F(0)$ from the space $L_{\xi, \beta}^{\infty}$ of sequences $f=\left(1, f_{1}\left(x_{1}\right), \ldots, f_{n}\left(x_{1}, \ldots, x_{n}\right), \ldots\right)$ of bounded functions $f_{n}\left(x_{1}, \ldots, x_{n}\right), f_{0} \equiv 1, n \geq 0$, that are defined on the phase space $\mathbb{R}^{n} \times\left(\mathbb{R}^{n} \backslash W_{n}\right)$, are invariant under permutations of the arguments $x_{i}, i=1, \ldots, n$, and are equal to zero on the set $W_{n}$. The norm in this space is defined by the formula

$$
\|f\|=\sup _{n \geq 0} \xi^{-n} \sup _{x_{1}, \ldots, x_{n}}\left|f_{n}\left(x_{1}, \ldots, x_{n}\right)\right| \exp \left\{\beta \sum_{i=1}^{n} \frac{p_{i}^{2}}{2}\right\},
$$


where $\xi, \beta$ are positive integers. Note that the sequences of $n$ particle equilibrium distribution functions of infinite systems belong to the space $L_{\xi, \beta}^{\infty}[1,10]$.

Let $Y \equiv\left\{x_{1}, \ldots, x_{s}\right\}, X \equiv\left\{Y, x_{s+1}, \ldots, x_{s+n}\right\}$, namely, $X \backslash Y=\left\{x_{s+1}, \ldots, x_{s+n}\right\}$, and let the symbol $|X|=|Y|+|X \backslash Y|=s+n$ denote the number of the elements of the set $X$. By the symbol $X_{Y}$ we denote the set $X$ with the subset $Y$ treated as a single element similar to $x_{s+1}, \ldots, x_{s+n}$. Let $L_{0}^{1}$ be the subspace of finite sequences of continuously differentiable functions with compact supports of space $L^{1}$ of sequences of integrable functions. For $F(0)$ from $L_{0}^{1}$, and hence for all $F(0) \in L_{0}^{1} \cap L_{\xi, \beta}^{\infty}$ it was proved in $[8,9]$ that the solution $F(t)=$ $\left(1, F_{1}\left(t, x_{1}\right), \ldots, F_{s}\left(t, x_{1}, \ldots, x_{s}\right), \ldots\right)$ of the initial value problem $(3),(4)$ is determined by the series expansion

$$
\begin{aligned}
F_{|Y|}(t, Y)= & \sum_{n=0}^{\infty} \frac{1}{n !} \int_{\mathbb{R}^{n} \times\left(\mathbb{R}^{n} \backslash W_{n}\right)} d(X \backslash Y) \sum_{\mathrm{P}: X_{Y}=\bigcup_{l} X_{l}}(-1)^{|\mathrm{P}|-1}(|\mathrm{P}|-1) ! \\
& \times \prod_{X_{l} \subset \mathrm{P}} S_{\left|X_{l}\right|}\left(-t, X_{l}\right) F_{|X|}(0, X), \quad|X \backslash Y| \geq 0,
\end{aligned}
$$

where $\sum_{\mathrm{P}}$ is the sum over all possible partitions $\mathrm{P}$ of the set $X_{Y}$ into $|\mathrm{P}|$ mutually disjoint nonempty subsets $X_{l} \subset X_{Y}, X_{k} \cap X_{l}=\varnothing, k \neq l$, such that the entire set $Y$ is contained in one of the subsets $X_{l}$.

On the set of sequences $f \in L_{0}^{1} \cap L_{\xi, \beta}^{\infty}$, the evolution operator $S_{\left|X_{l}\right|}\left(-t, X_{l}\right)$ from expansion (6) is given by the formula

$$
\begin{aligned}
& (S(-t) f)_{\left|X_{l}\right|}\left(X_{l}\right)=\left(S_{\left|X_{l}\right|}(-t) f_{\left|X_{l}\right|}\right)\left(X_{l}\right) \equiv S_{\left|X_{l}\right|}\left(-t, X_{l}\right) f_{\left|X_{l}\right|}\left(X_{l}\right) \\
& \quad= \begin{cases}f_{\left|X_{l}\right|}\left(\mathrm{x}_{1}\left(-t, X_{l}\right), \ldots, \mathrm{X}_{\left|X_{l}\right|}\left(-t, X_{l}\right)\right), & \text { if } x \in\left(\mathbb{R}^{\left|X_{l}\right|} \times\left(\mathbb{R}^{\left|X_{l}\right|} \backslash W_{\left|X_{l}\right|}\right)\right) \backslash \mathcal{M}_{\left|X_{l}\right|}^{0}, \\
0, & \text { if } x \in \mathbb{R}^{\left|X_{l}\right|} \times W_{\left|X_{l}\right|},\end{cases}
\end{aligned}
$$

where $\mathrm{X}_{1}\left(-t, X_{l}\right), i=1, \ldots,\left|X_{l}\right|$, is the solution of the initial value problem for the Hamilton equations of the system of $\left|X_{l}\right|$ particles with initial data $\mathrm{X}_{i}\left(0, X_{l}\right)=x_{i}\left(S_{\left|X_{l}\right|}(0)=I\right.$ is the identity operator). Under conditions (1) on the potential $\Phi$, the evolution operator (7) exists for $t \in(-\infty,+\infty)$; its properties are described in [1].

In the $n$th term of expansion (6), the form of the integrands is constructed by using the cumulant of order $1+n$ for the evolution operators $(7)$ :

$$
\sum_{\mathrm{P}: X_{Y}=\bigcup_{l} X_{l}}(-1)^{|\mathrm{P}|-1}(|\mathrm{P}|-1) ! \prod_{X_{l} \subset \mathrm{P}} S_{\left|X_{l}\right|}\left(-t, X_{l}\right) \equiv \mathfrak{A}_{1+|X \backslash Y|}\left(t, X_{Y}\right), \quad|X \backslash Y| \geq 0 .
$$

Here, the notation from formula (6) is used. Note that the order of the cumulant $\mathfrak{A}_{1+|X \backslash Y|}(t)$ is determined by the number of elements of the set $X_{Y}$ (in this case, by $1+|X \backslash Y|$ elements).

The simplest examples $(1+|X \backslash Y|=1,2,3)$ of the evolution operator $\mathfrak{A}_{1+n}(t)$ (8) have the form $[9,11,12]$

$$
\begin{aligned}
& \mathfrak{A}_{1}(t, Y)=S_{s}(-t, Y) \\
& \mathfrak{A}_{2}\left(t, Y, x_{s+1}\right)=S_{s+1}\left(-t, Y, x_{s+1}\right)-S_{s}(-t, Y) S_{1}\left(-t, x_{s+1}\right), \\
& \quad \mathfrak{A}_{3}\left(t, Y, x_{s+1}, x_{s+2}\right)=S_{s+2}\left(-t, Y, x_{s+1}, x_{s+2}\right)-S_{s+1}\left(-t, Y, x_{s+1}\right) S_{1}\left(-t, x_{s+2}\right) \\
& \quad-S_{s+1}\left(-t, Y, x_{s+2}\right) S_{1}\left(-t, x_{s+1}\right)-S_{s}(-t, Y) S_{2}\left(-t, x_{s+1}, x_{s+2}\right)+ \\
& \quad+2 ! S_{s}(-t, Y) S_{1}\left(-t, x_{s+1}\right) S_{1}\left(-t, x_{s+2}\right) .
\end{aligned}
$$

Thus, the cumulant representation of the solution (6) of the initial value problem for the BBGKY hierarchy (3), (4) is determined by the cumulants $\mathfrak{A}_{1+|X \backslash Y|}(t)$ (8) for the evolution operators $S_{\left|X_{l}\right|}\left(-t, X_{l}\right)(7)$. 


\section{Regularization of solution}

For $F(0) \in L_{\xi, \beta}^{\infty}$ every term $n \equiv|X \backslash Y|$ of expansion (6) contains divergent integrals with respect to the configuration variables. Let us show that the above-stated cumulant nature of the solution expansions (6) for the initial value problem of the BBGKY hierarchy (3), (4) guarantees the compensation of the divergent integrals, i.e., the cumulants are determined terms of expansion (6) as the sum of summands with divergent integrals that compensate one another. In order to prove this fact, let us rearrange the terms of expansion (6) so that they are represented by the simplest mutually compensating groups of summands. Such procedure will be called a regularization of the solution (6). In this case, the regularization will be based on expressing cumulants of higher order in terms of the first and second order cumulants. For fixed initial data the second-order cumulants will be determined by expressions which compensate each other over a certain bounded domain.

The next lemma shows that, in the general case, the cumulant of the $(1+n)$ th order $\mathfrak{A}_{1+n}(t)$, $n \geq 1$, is expressed via the first and second order cumulants.

Lemma 1. The equality

$$
\begin{aligned}
\mathfrak{A}_{1+|X \backslash Y|}\left(t, X_{Y}\right)= & \sum_{\substack{Z \subset X \backslash Y \\
Z \neq \varnothing}} \mathfrak{A}_{2}(t, Y, Z) \\
& \times \sum_{\mathrm{P}: X \backslash(Y \cup Z)=\bigcup_{l} X_{l}}(-1)^{|\mathrm{P}|}(|\mathrm{P}|) ! \prod_{X_{l} \subset \mathrm{P}} \mathfrak{A}_{1}\left(t, X_{l}\right), \quad|X \backslash Y| \geq 1,
\end{aligned}
$$

is true, where $\sum_{Z}$ is the sum over all the nonempty subsets $Z$ of the set $X \backslash Y, Z \subset X \backslash Y$, and the group of $|Z|$ particles evolves as a single element, and $\sum_{P}$ is the sum over all possible partitions $\mathrm{P}$ of the set $X \backslash(Y \cup Z)$ into $|\mathrm{P}|$ mutually disjoint nonempty subsets $X_{l} \subset X \backslash(Y \cup Z)$, $X_{k} \cap X_{l}=\varnothing, k \neq l$, such that every cluster of $\left|X_{l}\right|$ particles evolves as a single element.

The proof of Lemma 1 is based on the verification that (9) is equal to expression (8) by taking into account the representation of a second order cumulant in terms of the first order ones.

By using Lemma 1, represent integrands of every summand from expansion (6) in terms of the first and second order cumulants. As a result, expansion (6) for the solution of the initial value problem (3), (4) takes the form

$$
\begin{aligned}
& F_{|Y|}(t, Y)=\mathfrak{A}_{1}(t, Y) F_{|Y|}(0, Y)+\sum_{n=1}^{\infty} \frac{1}{n !} \int_{\substack{\mathbb{R}^{n} \times\left(\mathbb{R}^{n} \backslash W_{n}\right) \\
\mathrm{P}: X \backslash(Y \cup Z)=\bigcup_{l} X_{l}}} d(X \backslash Y) \\
& \times \sum_{\substack{Z \subset X \backslash Y \\
Z \neq \varnothing}} \mathfrak{A}_{2}(t, Y, Z) \sum_{X_{l} \subset \mathrm{P}}(-1)^{|\mathrm{P}|}|\mathrm{P}| ! \prod_{1} \mathfrak{A}_{1}\left(t, X_{l}\right) F_{|X|}(0, X), \quad|X \backslash Y| \geq 1 .
\end{aligned}
$$

For the initial data $F(0) \in L^{1} \cap L_{\xi, \beta}^{\infty}$, the equality

$$
\begin{aligned}
& \int_{\mathbb{R}^{n} \times\left(\mathbb{R}^{n} \backslash W_{n}\right)} d(X \backslash Y) \sum_{\substack{Z \subset X \backslash Y \\
Z \neq \varnothing}} \mathfrak{A}_{2}(t, Y, Z) \sum_{\mathrm{P}: X \backslash(Y \cup Z)=\bigcup_{l} X_{l}}(-1)^{|\mathrm{P}|}|\mathrm{P}| ! \prod_{X_{l} \subset \mathrm{P}} \mathfrak{A}_{1}\left(t, X_{l}\right) F_{|X|}(0, X) \\
= & \int_{\mathbb{R}^{n} \times\left(\mathbb{R}^{n} \backslash W_{n}\right)} d(X \backslash Y) \sum_{\substack{Z \subset X \backslash Y \\
Z \neq \varnothing}}(-1)^{|X \backslash(Y \cup Z)|_{\mathfrak{A}_{2}}(t, Y, Z) F_{|X|}(0, X), \quad|X \backslash Y| \geq 1,}
\end{aligned}
$$


is true. Here, we have used the Liouville theorem [1] and taken into account the relation

$$
\sum_{k=1}^{m}(-1)^{k} k ! s(m, k)=(-1)^{m}, \quad m \geq 1,
$$

where $s(m, k)$ is the Stirling number of the second kind defined as the number of all distinct partitions of a set containing $m$ elements into $k$ subsets.

Thus, by virtue of equality (11), the expansion (10) for the solution of the initial value problem for the BBGKY hierarchy (3), (4) takes the form

$$
\begin{aligned}
& F_{|Y|}(t, Y)=\mathfrak{A}_{1}(t, Y) F_{|Y|}(0, Y) \\
& +\sum_{n=1}^{\infty} \frac{1}{n !} \int_{\mathbb{R}^{n} \times\left(\mathbb{R}^{n} \backslash W_{n}\right)} d(X \backslash Y) \sum_{\substack{Z \subset X \backslash Y \\
Z \neq \varnothing}}(-1)^{|X \backslash(Y \cup Z)|} \mathfrak{A}_{2}(t, Y, Z) F_{|X|}(0, X), \quad|X \backslash Y| \geq 1
\end{aligned}
$$

where the notation from (9) is used.

Representation (13) will be called a regularized cumulant representation of the solution of the initial value problem for the BBGKY hierarchy (3), (4).

\section{Existence theorem}

Taking into account the invariance of the Gibbs distributions (the Maxwellian distribution can be extended to become a Gibbs distribution) with respect to the action of evolution operators $S(-t)(7)$ and using the condition (2) and the relation (9) we estimate the integrands in expansion (13).

Lemma 2. If $F(0) \in L_{\xi, \beta}^{\infty}$ then the inequality

$$
\begin{aligned}
& \left|\sum_{\substack{Z \subset X \backslash Y \\
Z \neq \varnothing}}(-1)^{|X \backslash(Y \cup Z)|} \mathfrak{A}_{2}(t, Y, Z) F_{|X|}(0, X)\right| \\
& \quad \leq 2\|F(0)\|\left(\xi \mathrm{e}^{2 \beta b}\right)^{s}\left(\xi \mathrm{e}^{2 \beta b}\right)^{n} \exp \left\{-\beta \sum_{i=1}^{s+n} \frac{p_{i}^{2}}{2}\right\}
\end{aligned}
$$

holds, where the notation from formulae (2), (5) and (9) is used.

As a consequence of Lemma 2, the following existence theorem is true.

Theorem. If $F(0) \in L_{\xi, \beta}^{\infty}$ is a sequence of nonnegative functions, then for $\xi<\frac{\mathrm{e}^{-2 \beta b-1}}{2 \tilde{C}_{1}} \sqrt{\frac{\beta^{\prime \prime}}{2 \pi}}$ and $t \in\left[0, t_{0}\right)$, where $t_{0}=\frac{1}{\tilde{C}_{2}}\left(\frac{\mathrm{e}^{-2 \beta b-1}}{2 \xi} \sqrt{\frac{\beta^{\prime \prime}}{2 \pi}}-\tilde{C}_{1}\right), \tilde{C}_{1}=\max (2 R, 1), \tilde{C}_{2}=\max \left(2(4 b+1), \frac{2}{\beta^{\prime}}\right)$, $\beta=\beta^{\prime}+\beta^{\prime \prime}, b \equiv \sup _{q \in[\sigma, R]}|\Phi(q)|\left(\left[\frac{R}{\sigma}\right]\right)$ and $\left[\frac{R}{\sigma}\right]$ is the integer part of the number $\frac{R}{\sigma}$, there exists a unique weak solution of the initial value problem for the BBGKY hierarchy (3), (4), namely, the sequence $F(t) \in L_{\xi, \beta}^{\infty}$ of nonnegative functions $F_{s}(t)$ determined by expansion (13).

Proof. Let particles interact via a short-range pair potential that satisfies conditions (1). We assume that, at the initial instant, the configuration coordinates $q_{i}, i=1,2, \ldots, s$, of the particles constituting the cluster $Y$ take values in a compact set of those $|Y|$ intervals $l_{i}$ with length $\left|l_{Y}\right|$ 
that $q_{i} \in l_{i}$. Then if during the time interval $[0, t)$ none of the particles of any cluster $Z \subset X \backslash Y$ interacts with the particles of cluster $Y$, then the operator equality holds

$$
S_{|Y \cup Z|}(-t, Y, Z)=S_{|Y|}(-t, Y) S_{|Z|}(-t, Z),
$$

and, as a result, we have

$$
\mathfrak{A}_{2}(t, Y, Z) F_{|X|}(0, X)=0 .
$$

Therefore, in this case, the integrands in the $n$th term of expansion (13) are equal to zero. Since $Z \subset X \backslash Y$, it follows that in the expansion (13) the domain $\mathbb{R}^{n} \backslash W_{n}$ of integration with respect to the configuration variables is determined by $n$ bounded intervals where the particles of the cluster $Y$ during the time interval $[0, t)$ interact with particles of the cluster $Z$ that contains the maximal number of particles, namely, with the particles of the cluster $X \backslash Y$. Thus, the domain $\mathbb{R}^{n} \backslash W_{n}$ of integration estimates the following finite value

$$
V(t) \leq\left(C+C_{0} t+\left(C_{1}+C_{2} t\right) n+t \sum_{i=s+1}^{s+n} p_{i}^{2}\right)^{n}
$$

where $C \equiv\left|l_{Y}\right|+2 s R, C_{0} \equiv 2 s(4 b+1)+\sum_{i=1}^{s} p_{i}^{2}, C_{1} \equiv 2 R$, and $C_{2} \equiv 2(4 b+1)$.

In view of estimates (14) and (15), we obtain

$$
\begin{aligned}
& \left|F_{|Y|}(t, Y)\right| \leq 2\|F(0)\|\left(\xi \mathrm{e}^{2 \beta b}\right)^{s} \exp \left\{-\beta \sum_{i=1}^{s} \frac{p_{i}^{2}}{2}\right\} \sum_{n=0}^{\infty} \frac{1}{n !}\left(\xi \mathrm{e}^{2 \beta b}\right)^{n} \\
& \quad \times \int_{\mathbb{R}^{n}} d p_{s+1} \cdots d p_{s+n} \exp \left\{-\beta \sum_{i=s+1}^{s+n} \frac{p_{i}^{2}}{2}\right\}\left(C+C_{0} t+\left(C_{1}+C_{2} t\right) n+t \sum_{i=s+1}^{s+n} p_{i}^{2}\right)^{n} .
\end{aligned}
$$

Taking in account the relation

$$
\begin{aligned}
\left(\left(C+C_{0} t\right)+\left(C_{1}+C_{2} t\right) n+t \sum_{i=s+1}^{s+n} p_{i}^{2}\right)^{n} \\
\quad=\sum_{k=0}^{n} \frac{n !}{k !}\left(C+C_{0} t\right)^{k} \sum_{r=0}^{n-k} \frac{1}{r !}\left(\left(C_{1}+C_{2} t\right) n\right)^{r} \frac{1}{(n-k-r) !} t^{n-k-r}\left(\sum_{i=s+1}^{s+n} p_{i}^{2}\right)^{n-k-r}
\end{aligned}
$$

and the inequality

$$
\left(\sum_{i=s+1}^{s+n} p_{i}^{2}\right)^{n-k-r} \exp \left\{-\beta^{\prime} \sum_{i=s+1}^{s+n} \frac{p_{i}^{2}}{2}\right\} \leq(n-k-r) !\left(\frac{2}{\beta^{\prime}}\right)^{n-k-r},
$$

we compute the integrals with respect to the momentum variables in the right-hand side of (16):

$$
\int_{\mathbb{R}^{n}} d p_{s+1} \cdots d p_{s+n} \exp \left\{-\beta^{\prime \prime} \sum_{i=s+1}^{s+n} \frac{p_{i}^{2}}{2}\right\}=\left(\frac{2 \pi}{\beta^{\prime \prime}}\right)^{\frac{n}{2}}, \quad \beta=\beta^{\prime}+\beta^{\prime \prime} .
$$

Then estimate (16) takes the form

$$
\left|F_{|Y|}(t, Y)\right| \leq 2\|F(0)\|\left(\xi \mathrm{e}^{2 \beta b}\right)^{s} \exp \left\{-\beta \sum_{i=1}^{s} \frac{p_{i}^{2}}{2}\right\}
$$




$$
\times \sum_{n=0}^{\infty}\left(2 \xi \mathrm{e}^{2 \beta b}\right)^{n}\left(\frac{2 \pi}{\beta^{\prime \prime}}\right)^{\frac{n}{2}} \sum_{k=0}^{n} \frac{\left(C+C_{0} t\right)^{k}}{k !} \sum_{r=0}^{n-k} \frac{n^{r}}{r !}\left(C_{1}+C_{2} t\right)^{r} \frac{t}{(n-k-r) !} .
$$

Let us put $\tilde{C}_{1}=\max \left(C_{1}, 1\right)$ and $\tilde{C}_{2}=\max \left(C_{2}, \frac{2}{\beta^{\prime}}\right)$ and continue estimate $\left(16^{\prime}\right)$. For arbitrary $t \geq 0$, the inequalities $\tilde{C}_{1}+\tilde{C_{2}} t \geq 1$ and $\left(\tilde{C_{1}}+\tilde{C}_{2} t\right) \frac{2 t}{\beta^{\prime}} \geq 1$ are true, and, therefore,

$$
\left(C_{1}+C_{2} t\right)^{r}\left(\frac{2 t}{\beta^{\prime}}\right)^{n-k-r} \leq\left(\tilde{C}_{1}+\tilde{C}_{2} t\right)^{n}
$$

By using the inequalities $\sum_{r=0}^{n-k} \frac{n^{r}}{r !} \leq \mathrm{e}^{n}$ and $\sum_{k=0}^{n} \frac{\left(C+C_{0} t\right)^{k}}{k !} \leq \mathrm{e}^{\left(C+C_{0} t\right)}$ and taking estimate $\left(16^{\prime}\right)$ into account, we obtain

$$
\begin{aligned}
\left|F_{|Y|}(t, Y)\right| \leq & 2\|F(0)\|\left(\xi \mathrm{e}^{2 \beta b}\right)^{s} \exp \left\{-\beta \sum_{i=1}^{s} \frac{p_{i}^{2}}{2}\right\} \\
& \times \mathrm{e}^{\left(C+C_{0} t\right)} \sum_{n=0}^{\infty}\left(2 \xi \mathrm{e}^{2 \beta b+1} \sqrt{\frac{\beta^{\prime \prime}}{2 \pi}}\right)^{n}\left(\tilde{C}_{1}+\tilde{C}_{2} t\right)^{n} .
\end{aligned}
$$

Thus, if $\xi<\frac{\mathrm{e}^{-2 \beta b-1}}{2 \tilde{C}_{1}} \sqrt{\frac{\beta^{\prime \prime}}{2 \pi}}$ then series (19) converges for $0 \leq t<t_{0} \equiv \frac{1}{\tilde{C}_{2}}\left(\frac{\mathrm{e}^{-2 \beta b-1}}{2 \xi} \sqrt{\frac{\beta^{\prime \prime}}{2 \pi}}-\tilde{C}_{1}\right)$. We have thus shown that, under the conditions of the theorem, series (16) converges.

Finally, using the well-known theorems of functional analysis [13] and arguing similarly to $[1,2]$, we show that the sequence $F(t)$ is the unique weak solution of the initial value problem for the BBGKY hierarchy (3), (4).

\section{Conclusion}

The theory of the BBGKY hierarchy is developing now since the area of its application grows [14, 15]. At present while solving the initial value problem of the BBGKY hierarchy, many mathematical problems arise $[3,5,16,17]$. In this paper one of such problems is considered for infinite particle system employing the method of the interaction region [5]. Taking into account the cumulant representation $[8,9]$ we construct a new regularized representation of the solution of the BBGKY hierarchy for a one-dimensional infinite system of hard spheres interacting via a short-range potential. For the initial data from the space of sequences of functions which are bounded in configuration variables and exponentially decreasing in momentum variables, existence of the cumulant representation allows to properly regularize such expansion for the solution, determining by second order cumulant as the sum of summands with divergent integrals that compensate one another. A multidimensional case and a case of a more general interaction potential will be investigated in further contribution.

\section{Acknowledgement}

The author is pleased to thank Professor Victor Gerasimenko for many useful discussions and is grateful to the referees for helpful comments and references. This work was partially supported by the National Academy of Sciences of Ukraine through grant No 0105 U005666 for young scientists and partially supported by INTAS, grant No 00-0015. 
[1] Petrina D.Ya., Gerasimenko V.I., Malyshev P.V., Mathematical foundations of classical statistical mechanics. Continuous systems, 2nd ed., London - New York, Taylor \& Francis Inc., 2002.

[2] Cercignani C., Gerasimenko V.I., Petrina D.Ya., Many-particle dynamics and kinetic equations, Kluwer Acad. Publ., 1997.

[3] Cercignani C., Illner R., Pulvirenti M., The mathematical theory of dilute gases, Applied Mathematical Sciences, Vol. 106, New York, Springer, 1994.

[4] Spohn H., Large scale dynamics of interacting particles, Springer, 1991.

[5] Petrina D.Ya., Mathematical description of the evolution of infinite systems of classical statistical physics. I. Locally perturbed one-dimensional systems, Teoret. Mat. Fiz., V.38, 1979, 230-262 (in Russian).

[6] Petrina D.Ya., Gerasimenko V.I., Mathematical description of the evolution of the state of infinite systems of classical statistical mechanics, Uspekhi Mat. Nauk, 1983, V.38, 3-58 (in Russian).

[7] Gerasimenko V.I., Ryabukha T.V., Dual nonequilibrium cluster expansions, Dopov. Nats. Akad. Nauk Ukr. Mat. Prirodozn. Tekh. Nauki, 2003, N 3, 16-22 (in Ukrainian).

[8] Gerasimenko V.I., Ryabukha T.V., Stashenko M.O., On the BBGKY hierarchy solutions for many-particle systems with different symmetry properties, in Proceedings of Fifth International Conference "Symmetry in Nonlinear Mathematical Physics" (June 23-29, 2003, Kyiv), Editors A.G. Nikitin, V.M. Boyko, R.O. Popovych and I.A. Yehorchenko, Proceedings of Institute of Mathematics, Kyiv, 2004, V.50, Part 3, 1308-1313.

[9] Gerasimenko V.I., Ryabukha T.V., Cumulant representation of solutions of the Bogolyubov chains of equations, Ukrain. Mat. Zh., 2002, V.54, 1313-1328 (English transl.: Ukrainian Math. J., 2002, V.54, 1583-1601).

[10] Ruelle D., Statistical mechanics. Rigorous results, New York - Amsterdam, W.A. Benjiamin Inc., 1969.

[11] Cohen E.G.D., Cluster expansions and the hierarchy. I. Non-equilibrium distribution functions, Physica, 1962, V.28, 1045-1059.

[12] Green H.S., Piccirelli R.A., Basis of the functional assumption in the theory of the Boltzmann equation, Phys. Rev. (2), 1963, V.132, 1388-1410.

[13] Reed M., Simon B., Methods of modern mathematical physics. Vol. 1: Functional analysis, New York London, Academic Press, 1972.

[14] Kaniadakis G., BBGKY hierarchy underlying many-particle quantum mechanics, Phys. Lett. A, 2003, V.310, 377-382, quant-ph/0303159.

[15] Tarasov V.E., Fractional systems and fractional Bogoliubov hierarchy equations, Phys. Rev. E, 2005, V.71, 011102, 12 pages, cond-mat/0505720.

[16] Illner R., Pulvirenti M., Global validity of the Boltzmann equation for a two-dimensional rare gas in vacuum, Comm. Math. Phys., 1986, V.105, 189-203.

[17] Illner R., Pulvirenti M., A derivation of the BBGKY-hierarchy for hard sphere particle systems, Transport Theory Statist. Phys., 1987, V.16, 997-1012. 